\title{
Plasma frio atmosférico - novas oportunidades de pesquisa numa plataforma versátil e portadora de futuro
}

Clodomiro Alves Junior ${ }^{1}$

\author{
${ }^{1}$ Labplasma - Laboratório de Plasma Aplicado na Agricultura, Saúde e Meio Ambiente, Universidade Federal Rural do \\ Semi-árido, Mossoró, RN, Brasil. \\ e-mail: clodomiro.jr@ufersa.edu.br
}

\section{INTRODUÇÃO}

Apesar de ocupar mais de $99 \%$ do espaço cósmico, ele é visto aqui na terra em raras situações, como por exemplo na aurora boreal. Este fenômeno que acontece nos polos, origina-se quando elétrons vindos do vento solar são acelerados pela força magnética terrestre e colidem com átomos da atmosfera. Dessa interação, uma cascata de eventos, dentre as quais a ionização e excitação dos átomos, formam uma "salada" de espécies em diferentes estados energéticos. Essa "salada" de espécies, fora do equilíbrio termodinâmico e energeticamente disponível para recombinar-se com a vizinhança, foi apresentado em 1928 como o quarto estado da matéria e denominado de plasma [1]. Entretanto, somente após a segunda guerra mundial foi que pesquisadores começaram a se interessar pela formação de plasma artificial e seus benefícios potenciais para a humanidade. No início houve a corrida por plasmas para fusão termonuclear, em que plasma era produzido em baixíssima pressão para a fusão nuclear controlada usando fortes campos magnéticos [2]. Depois, na década de 70, iniciaram pesquisas mais intensas em tecnologias a plasma, não apenas na indústria eletrônica, mas também nas indústrias aeroespacial, automotiva, metalúrgica, siderúrgica, biomédica, têxtil, óptica e de papel [3-10]. Essas técnicas, em sua grande maioria, utilizavam plasma frio de baixa pressão - plasma em que a energia do elétron é muito maior que a energia média das demais partículas do plasma - enquanto aplicações como a siderúrgica, utilizavam plasma térmico - em que o sistema está próximo ao equilíbrio, ou seja, a energia do elétron é aproximadamente a mesma que das demais espécies. Essas tecnologias de plasma frio possuem limitação de uso devido à baixa pressão necessária para produção do plasma. Além da limitação dimensional, estão outras como a necessidade do produto a ser tratado possuir baixa pressão de vapor e assim manter sua integridade durante o processamento. Uma tecnologia que possa ser usada na pressão atmosférica e que mantenha o plasma frio, ou seja, a característica de não equilíbrio que permite colisões de altas energia entre elétrons e as demais espécies, mantendo o ambiente em baixa temperatura. Uma tecnologia como essa é altamente atrativa em aplicações de materiais termicamente sensíveis como polímeros, líquidos e tecidos vivos [11,12]. Pesquisas dos últimos 20 anos estão sendo desenvolvidas e denominadas de plasma frio atmosférico (ou Cold Atmospheric Pressute Plasma PFA). Elas direcionam para aplicações na área de saúde como cicatrização de feridas, coagulação sanguínea, desinfecção de cárie dentária e alteração de funções de células de mamíferos com potencial para novas terapias de cancro [13-17]. Na agricultura pode ser utilizado para estimular o crescimento de plantas e reduzir patógenos, germinação de sementes, descontaminação de superfícies biologicamente ativas de frutos e descontaminação pós-colheita [18-23]. Também na área ambiental, onde pode ser utilizado para descontaminação de ambientes, líquidos e sólidos, tratamento de água, degradação de corantes, entre outros [24,25]. No Brasil a técnica ainda é pouco utilizada e difundida. Alguns centros de pesquisa que utilizam, a fazem de maneira isolada e pouco sistemática. Buscas no diretório de pesquisa do CNPq realizadas em 02/08/2020, indicam que 10 grupos de pesquisas no Brasil existem com a palavra "plasma" em seu nome e, desses, apenas 02 grupos de pesquisa possui o termo "plasma atmosférico" ou "plasma frio" em seu nome. A Universidade Federal Rural do Semi-árido - UFERSA vem desenvolvendo pesquisas na área de plasma frio atmosférico aplicado na agricultura, saúde e meio ambiente desde 2012, onde estão sendo obtidos interessantes e inéditos resultados. Considerando o baixo custo e relevância da pesquisa, bem como seu caráter multidisciplinar, inovador e de integração inter-departamental, a divulgação dessa tecnologia poderá ser um importante passo na disseminação em outras instituições de pesquisas e nas indústrias nacionais. Com nossa experiência adquirida nos últimos 8 anos, será possível uma abordagem sobre principais fontes de tensão, configurações de reatores e resultados de aplicações na agricultura, saúde e 
meio ambiente.

\section{MATERIAIS E MÉTODOS}

Devido às altas taxas de colisões em pressões atmosféricas, há forte tendência ao equilíbrio térmico das espécies do plasma. A esse plasma denominamos de plasma térmico e que não faz parte do escopo deste trabalho. Para criar um plasma de não-equilíbrio, ou plasma frio em pressão atmosférica, a maneira mais viável é "bombeando" seletivamente os elétrons, de forma a aumentar a diferença entre a temperatura eletrônica e do gás. A densidade da potência de alimentação influencia muito o estado de equilíbrio termodinâmico (LTE) do plasma. No geral, uma alta potência induz plasmas térmico (por exemplo, plasmas de arco), enquanto plasmas não-LTE são favorecidos por uma baixa densidade de potência de alimentação ou por uma fonte de energia pulsada. Neste último caso, a curta duração do pulso impede que o estado de equilíbrio se estabeleça. Portanto, é possível produzir plasma frio mesmo em pressões altas. Para isso, várias estratégias são utilizadas visando evitar centelhas ou arcos, estabilizando a descarga e assim produzir plasmas frios em altas pressões.

\subsection{Descarga corona}

São descargas elétricas de potência relativamente baixa, que ocorrem na pressão atmosférica ou próximo a ela. Uma coroa, daí o nome corona, é invariavelmente gerada por fortes campos elétricos associados a fios de diâmetro pequeno, agulhas ou bordas afiadas de um eletrodo [26]. A coroa aparece como uma fraca descarga filamentar irradiando para fora do eletrodo de descarga. Devido à baixa potência, ela pode ser produzida por fonte de alimentação DC ou DC pulsada, sem aparecimento de arcos. De um modo geral, nossos experimentos com descarga corona foram realizados no arranjo experimental como ilustrado na Figura $1 \mathrm{~A}$. A fonte de tensão pulsada era variável entre 1 a $10 \mathrm{kV}$ e frequência de repetição de $500 \mathrm{~Hz}$ a $3 \mathrm{KHz}$, ajustadas de acordo com a aplicação. Valores da tensão medida entre eletrodos e da corrente que passava através de um capacitor em série com a carga eram utilizadas para determinar a energia e potência do plasma. As espécies do plasma foram analisadas por espectroscopia de emissão óptica, a partir do espectro transmitido por uma fibra óptica colocada a $5 \mathrm{~mm}$ da descarga. Uma agulha de seringa de aço inoxidável $(1.20 \mathrm{~mm}$ x $40.0 \mathrm{~mm}) \mathrm{com}$ uma ponta cônica de raio de curvatura de $0,27 \mathrm{~mm}$ foi utilizado como eletrodo. Investigou-se com esse tipo de descarga (a) efeito do plasma sobre água destilada[31]; (b) degradação de alaranjado de metila por plasma corona [32] (c) efeito do plasma sobre uma superfície de água-mãe (resíduo de água hipersalina resultante da produção do sal marinho) [33-35]; (d) produção de nanopartículas de Ag a partir da interação do plasma com solução $2 \mathrm{mM}$ de nitrato de prata [36]; As condições experimentais de (a) e (b) foram as mesmas, ou seja, utilizou-se $15 \mathrm{kV}$ e $500 \mathrm{~Hz}$, com polarização anódica e catódica. O efeito sobre água-mãe foi realizado num recipiente fechado, contendo Hélio, com polarização catódica (Fig. 1B, superior) e anódica (Fig. 1B, inferior), usando voltagem de $2,3 \mathrm{kV}$ e frequência de repetição de pulso de $1 \mathrm{kHz}$. Para a produção de nanopartículas de Ag, utilizou-se uma voltagem de $7,6 \mathrm{kV}$ e $588 \mathrm{~Hz}$.

Figura 1: (A) Ilustração do arranjo experimental utilizado para investigação de plasma produzido por descarga corona em diferentes superficies; (B) Ilustração de descarga corona sobre água-mãe, com polarização catódica (acima) e anódica (abaixo).

(A)

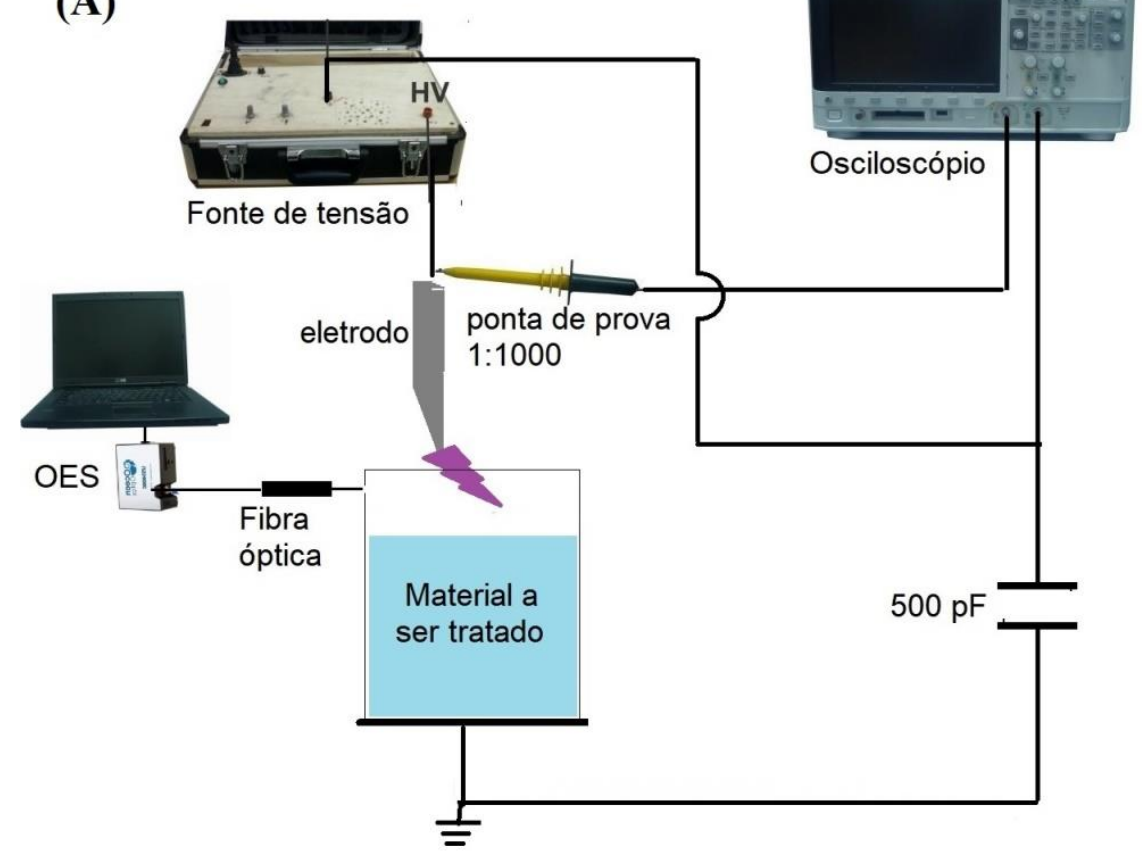

(B)
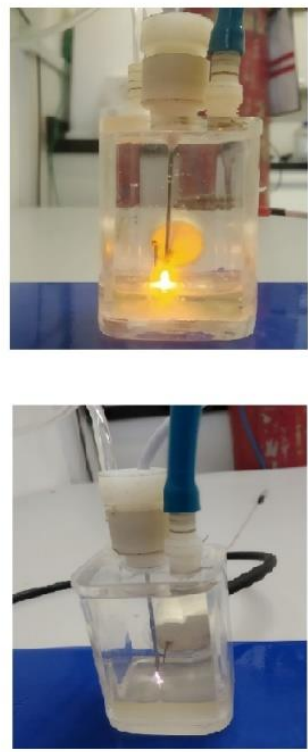


\subsection{Descarga por barreira dielétrica (DBD)}

Um sistema genérico consiste em dois eletrodos metálicos, em que pelo menos um desses eletrodos é coberto por uma camada dielétrica (Fig. 2). Para garantir uma operação estável do plasma, o espaço que separa os eletrodos é limitado a alguns milímetros de largura. A descarga é iniciada por meio de uma fonte de energia senoidal [27] ou pulsada [28]. Dependendo da composição do gás de trabalho, excitação, tensão e frequência, a descarga pode ser filamentar (indesejável) ou difusa [29]. A escolha do material da barreira é muito importante devido aos efeitos de carregamento, efeitos de ionização por penetração em superfícies, entre outros. $\mathrm{O}$ desempenho da barreira também depende do gás e da frequência do campo. A formação de descargas uniformes, do tipo difusa, é baseada em carga superficial suficiente do dielétrico e em numerosas rupturas sobrepostas. As centelhas que se expandem radialmente, fornecem carga espacial necessária para a autoestabilização de um plasma semelhante ao luminescente (glow discharge) [30]. Como é de se esperar, um fator importante no desempenho da descarga é a constante dielétrica da barreira. Em nossos experimentos foi utilizado um sistema DBD simples, construído de fenolite dupla face, com eletrodos alternados, construídos com auxílio da erosão química do revestimento de cobre nas duas faces do fenolite, conforme ilustrado na figura 2. Observe que os eletrodos estão dispostos num mesmo plano, criando um plasma de superfície. Esse sistema foi utilizado para experimentos de (a) inativação de micro-organismos; (b) modificação de superfície de quitosana e de cera de carnaúba. Foram utilizados diferentes tipos de fungos extraídos de sementes de mulungú (E. velutina), a saber: A. niger, A. flavus, Fusarium sp., Brachysporium sp. e Rhizopus sp. Nos esporos desses fungos foi aplicado descarga DBD de $9 \mathrm{kV}$ e $640 \mathrm{~Hz}$, usando atmosfera de ar atmosférico ou de hélio, em tempos de 180, 360 e $540 \mathrm{~s}$ [37]. Também foi utilizado para inativação de esporos de bactéria $E$ coli e de fungo C. albicans [38]. Para esses últimos foi utilizado uma voltagem de $10,3 \mathrm{kV}$ e frequência de 4 $\mathrm{kHz}$, com tempos de tratamento de 450, 900 e $1350 \mathrm{~s}$. Outras configurações de plasma DBD também foram utilizadas para tratamento de líquidos e para produção de jatos de plasma (Fig. 2, C-D). Nessa configuração, o material a ser tratado fica entre os dois eletrodos e por isso dizemos que é uma descarga DBD de volume. Com pequenas adaptações do aparato da figura 2D, pode-se construir um jato de plasma (Fig. 2E), tomando o cuidado de que o eletodo externo esteja aterrado. Para que o plasma gerado possua maior alcance, gases inertes como He ou Ar são utilizados. Este último exemplo possui uma aplicação crescente na medicina, para cicatrização de cortes e de feridas, descamação (peeling), remoção de cáries, doenças inflamatórias da pele, inclusive tratamento de câncer [10-17].

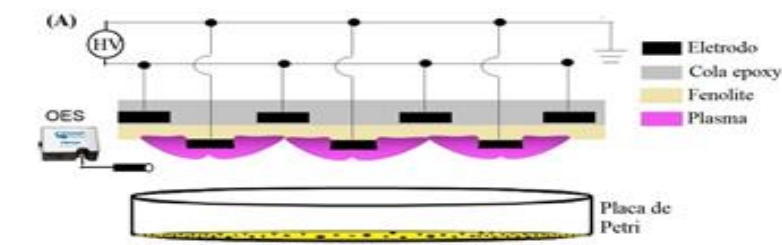

(B)
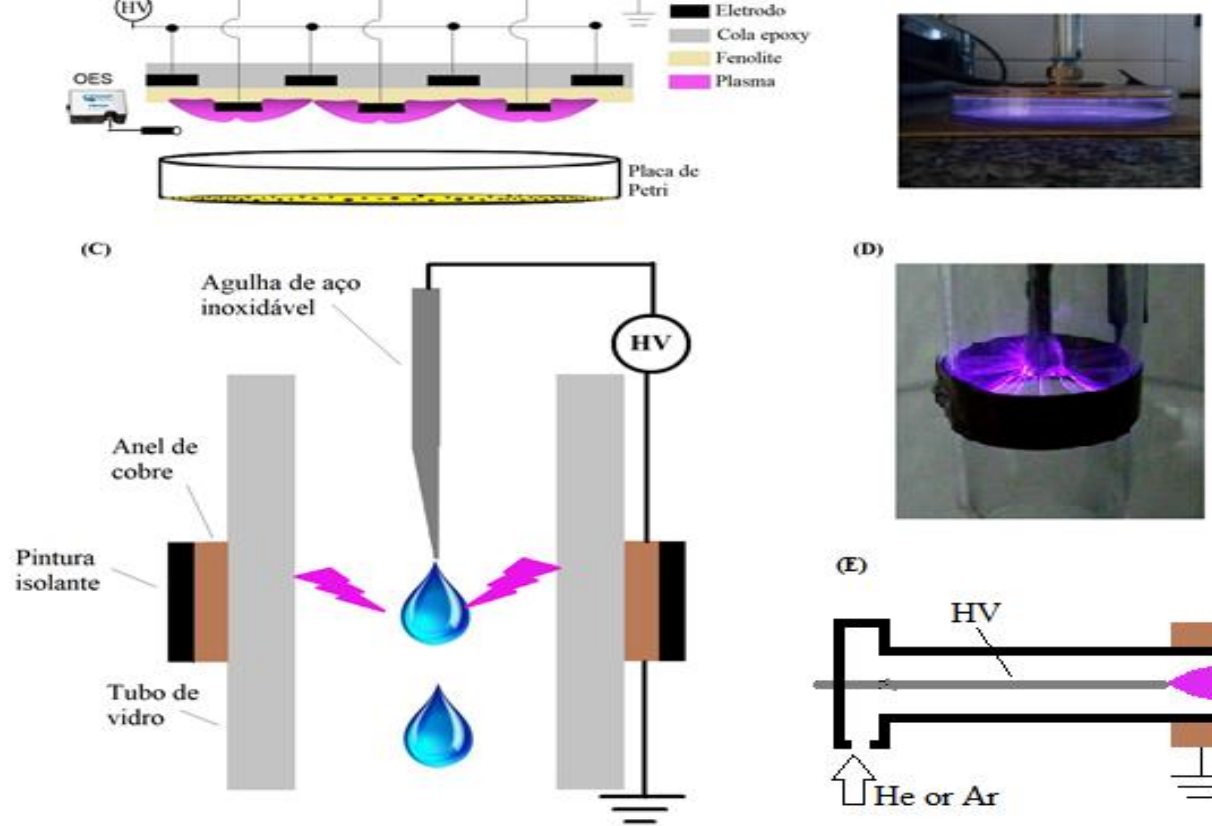

(D)

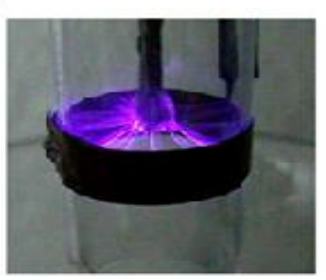

(E)

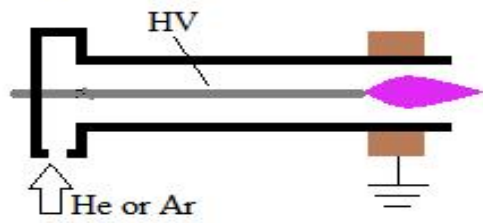

Figure 2: Configurações de reatores usando descarga por barreira dielétrica (DBD). (A) Detalhes de construção do um reator DBD de superfície; (B) foto da aplicação do plasma DBD de superfície sobre fungos; (C) Detalhes de construção de reator DBD de volume; (D) foto descarga sobre uma gota d'água, durante tratamento de água por gotejamento e (E) Desenho esquemático de jato de plasma. 


\section{RESULTADOS E DISCUSSÃO}

Os resultados aqui apresentados serão mais ilustrativos da versatilidade e eficiência do plasma na modificação de superfície, inclusive superfícies líquidas, voláteis e tecidos vivos, como é o caso do plasma frio atmosférico, tema do presente trabalho. Nossos resultados estão direcionados para aplicações na agricultura, saúde e meio ambiente, porque eram esses os principais campos de aplicações não contemplados pelo plasma de baixa pressão. Na agricultura e saúde isso acontecia pela vulnerabilidade do tecido vivo à baixa pressão ou termo sensibilidade. No caso de meio ambiente, porque os sistemas de vácuo limitavam o uso e pelo fato de muito dos estados físicos serem líquidos. Aplicações na agricultura consistiram em tratamento de sementes para quebra da dormência, germinação e vigor das plântulas. Foram utilizadas sementes do semiárido (mulungú, leucena, sabiá, umbu e pepaconha) que tinham alta dormência. Testes de molhabilidade mostraram que todas as sementes reduziram o ângulo de contato e, consequentemente, capacidade de embebição. Também aumento significativo da taxa de germinação e vigor. Algumas pesquisas estão sendo realizadas para compreender melhor o caminho da água nessas sementes e como o plasma altera a fisiologia da germinação da semente [39-42]. Verificou-se ainda que sementes saudáveis e sem dormência, quando regadas com água tratada por plasma, apresentavam uma taxa de germinação e vigor superiores àquelas regadas com água sem tratamento. Esse último resultado motivou a realização de vários experimentos de plasma aplicado em água, com diferentes salinidades. Água destilada $(\mathrm{pH} \mathrm{6,8)} \mathrm{gotejada}$ numa razão de 1 gota/s (Fig. 2), teve o $\mathrm{pH}$ reduzido para 4,85 quando submetido a uma descarga de picos de voltagem de $16,5 \mathrm{kV}$, repetidos em frequência de $15,5 \mathrm{kHz}$ [43]. Esse valor de $\mathrm{pH}$ foi linearmente decrescente com o aumento de voltagem, até valores de $28,5 \mathrm{kV}$, quando o valo do $\mathrm{pH}$ foi 3,08 . Também foram realizados experimentos de descarga corona sobre superfície de água destilada, tratada durante 15 minutos, usando voltagem de $15 \mathrm{kV}$ e frequência de $500 \mathrm{~Hz}$. Foi observado que imediatamente após o tratamento, o pH reduziu de 6,5 para 5,8. Uma vez que espécies à base de nitrogênio e oxigênio estão presentes num plasma de ar atmosférico, sua interação com água, podem levar à formação de espécies moleculares e radicais, como peróxido de hidrogênio $\left(\mathrm{H}_{2} \mathrm{O}_{2}\right)$, hidrogênio molecular $\left(\mathrm{H}_{2}\right)$, oxigênio molecular $\left(\mathrm{O}_{2}\right)$, radicais hidroxila $\left(\mathrm{OH}^{*}\right), \mathrm{NO}_{\mathrm{x}}$, entre outros. Elétrons podem ficar no estado hidratado (plasma catódico), reagindo com moléculas da água e promovendo a formação de hidroxilas, $\mathrm{H}^{*}, \mathrm{H}_{2} \mathrm{O}^{+}, \mathrm{H}_{2}$, entre outros. Esse sistema complexo da interação plasma-água ainda precisa ser mais compreendido para responder resultados como os apresentados aqui. A evolução temporal da água tratada por plasma foi analisada durante $48 \mathrm{~h}$, verificando-se que houve redução de $\mathrm{pH}$ enquanto a condutividade elétrica e concentração de nitrato foram aumentadas. Isso significa que houve aumento da concentração de $\mathrm{H}^{+}$em relação ao $\mathrm{OH}^{-}$, resultando em aumento da acidez da água, mesmo após o plasma ter sido aplicado. Uma hipótese para isso é que, após as espécies introduzidas na água pelo plasma, cascatas de reações devem ocorrer com moléculas do ar atmosférico, aumentando a acidez através da formação de $\mathrm{HNO}_{3}$. Esses resultados dão um bom exemplo da capacidade de transferência de espécies reativas do plasma para a água. Quando se aumentou a concentração de sal na água e sobre a mesma aplicou-se a descarga corona, verificou-se que o plasma induziu a precipitação na superfície da água. O filme salino formado possuía composições que variavam com as condições dos parâmetros de plasma utilizados. Outros resultados interessantes observados na interação de plasma com líquido foi a desinfecção de água, água de coco, leite e sucos, além da degradação de corantes. Um campo importante que estamos desenvolvendo atualmente é a modificação de superfícies de implantes por CAP. Foi observado que superfícies de titânio e de aços inoxidáveis quando tratado por plasma de ar, aumentam a molhabilidade, adesão celular e ação antimicrobiana. Inclusive quando estas superfícies estão lacradas com material polimérico, foi possível observar esse efeito. Isso significa que implantes podem ser tratados ainda na embalagem, para serem usados posteriormente.

\section{CONCLUSÕES}

Uma revisão sobre plasma frio atmosférico foi apresentada, contendo os principais resultados obtidos por um grupo de pesquisa da Universidade Federal Rural do Semi-árido (UFERSA). O artigo chama atenção para a versatilidade, simplicidade de uso e multidispliplinaridade da técnica, servindo como núcleo de integração interdepartamental institucional. A técnica teve início neste século e está sendo usada com sucesso em muitas aplicações da saúde, agricultura e meio ambiente. Uma área específica denominada Plasma Medicine, que já possui muitas soluções comerciais para cicatrização de cortes e feridas, entre outras doenças da pele, é um exemplo do crescimento e importância da técnica. Apesar das aplicações comerciais já existentes, muitas questões ainda precisam ser respondidas, as quais exigem um esforço multidisciplinar.

\section{AGRADECIMENTOS}

Este projeto foi financiado pelo Conselho Nacional de Desenvolvimento Científico e Tecnológico 
(306087/2013-8, 430863 / 2016-0) e Instituto Nacional de Engenharia de Superfícies (CNPq- 465423 / 20140) e Conselho Nacional de Melhoria do Ensino Superior (CAPES).

\section{BIBLIOGRAFIA}

[1] LANGMUIR, I., "Oscillations in ionized gases", Proc Natl Acad Sci., U S A. 1928 v.14, n. 8, pp. 627637, 1928.

[2] DAM, J., "Review of tokamak plasma heating by wave damping in the ion cyclotron range of frequency", Plasma Physics Controlled Fusion, v. 29, n. 443, 1987.

[3] ABD JELIL, R., "A review of low-temperature plasma treatment of textile materials", Journal of Materials Science, v. 50, pp. 5913-5943, 2015.

[4] PETLINA, D.G., TVERDOKHLEBOV, S.I., ANISSIMOV, Y.G., "Plasma treatment as an efficient tool for controlled drug release from polymeric materials: A review", Journal of Controlled Release, v.266, pp. 57-74, 2017.

[5] HEBERLEIN, J., MURPHY, A. B., “Thermal plasma waste treatment”, J. Phys. D: Appl. Phys. v. 41, 2008.

[6] LADIZESKY, N. H., WARD, I. M., "A review of plasma treatment and the clinical application of polyethylene fibres to reinforcement of acrylic resins", Journal of Materials Science: Materials in Medicine, v. 6, pp. 497 504, 1995.

[7] ALVES, C., DE ARAÚJO, O. F., DE SOUSA, M.R.R., "Comparison of plasma-assisted nitriding techniques. In: Wang Q.J., Chung YW. (eds) Encyclopedia of Tribology. Springer, Boston, MA., 2013.

[8] SHI, F.F., "Recent advances in polymer thin films prepared by plasma polymerization Synthesis, structural characterization, properties and applications", Surface and Coatings Technology, v. 82, pp. 1-15, 1996.

[9] RANDHAWA, H., "Review of plasma-assisted deposition processes", Thin Solid Films, v. 196, pp. 329349, 1991.

[10] CHU, P. K., "Applications of plasma-based technology to microelectronics and biomedical engineering”, Surface \& Coatings Technology, v. 203, pp. 2793-2798, 2009.

[11] FRIDMAN, G., FRIEDMAN, G., GUTSOL, A., et al., "Applied plasma medicine", Plasma Process. Polym., v. 5, pp. 503-533, 2008.

[12] KONG, M.G., KROESEN, G., MORFILL, G., et al., "Plasma medicine: an introductory review", New Journal of Physics,v. 11, n.11, pp. 115012, 2009.

[13] IZA, F., KIM, G. J., LEE, S. M.J., et al., "Microplasmas: Sources, particle kinetics, and biomedical applications", Plasma Processes and Polymers, v. 5, n. 4, pp. 322-344, 2008.

[14] WeLTMANN, K. D., KINDEL, E., VON WOEDTKE, T., et al., "Atmospheric-pressure plasma sources: prospective tools for plasma medicine”, Pure Applied Chemistry, v. 82, n. 6, 2010.

[15] WELTMANN, K. D., POLAK, M., MASUR, K., et al., "Plasma processes and plasma sources in medicine", Contributions to Plasma Physics, v. 52, n. 7, pp. 644-654, 2012.

[16] LAROUSSI, M., KONG, M. G., MORFILL, G., et al., "Plasma Medicine: Applications of LowTemperature Gas Plasmas in Medicine and Biology" Cambridge University Press, 2012.

[17] VON WOEDTKE, T., REUTER, S., MASUR, K., et al., "Plasmas for medicine", Physics Reports, v. 530, n. 4, pp. $291-320,2013$.

[18] SCHNABEL, U , POLAK, M., WINTER, J., et al., "Low temperature atmospheric pressure plasma sources for microbial decontamination", Journal of Physics D: Applied Physics, v. 44, n. 1, pp. 013002, 2011.

[19] RANDENIYA, L.K. , DE GROOT, G. J. J. B., "Non-Thermal Plasma Treatment of Agricultural Seeds for Stimulation of Germination, Removal of Surface Contamination and Other Benefits: A Review", Plasma Processes and Polymers, ppap.201500042, 2015.

[20] TAGHIZADEH, L., BRACKMAN, G., NIKIFOROV, A., et al., "Inactivation of Biofilms Using a Low Power Atmospheric Pressure Argon Plasma Jet the Role of Entrained Nitrogen", Plasma Processes and Polymers, v. 12, pp. 75-81, 2015. 
[21] EL SHAER, M., MOBASHER, M., ABDELGHANY, A., "Treatment of Microorganisms in Vegetables and Fruits by Gliding Arc", Plasma Medicine, v. 4, n. 1-4, pp. 57-65, 2014.

[22] MITSUGI, F., NAGATOMO, T., TAKIGAWA, K., et al., "Properties of Soil Treated With Ozone Generated by Surface Discharge”, IEEE Transactions on plasma science, v. 42, n.12, pp. 3706-3711, 2014.

[23] YUSAF, T., AL-JUBOORI, R. A., "Alternative methods of microorganism disruption for agricultural applications", Applied Energy, v.114, pp. 909-923, 2014.

[24] MALIK, M. A., GHAFFAR, A., MALIK, S. A.. "Water purification by electrical discharges", Plasma Sources Science and Technology, v.10, pp. 82-91, 2001.

[25] LOCKE, B. R., SHIH, K-Y., "Review of the methods to form hydrogen peroxide in electrical discharge plasma with liquid water", Plasma Sources Science and Technology, v. 20, 034006 (15pp), 2011.

[26] BÁRDOS, L., BARÁNKOVÁ, H., "Cold atmospheric plasma: Sources, processes, and applications", Thin Solid Films, v. 518, pp. 6705-6713, 2010.

[27] ROBERT, E., DARNY, T., DOZIAS, S., et al., "New insights on the propagation of pulsed atmospheric plasma streams: from single jet to multi jet arrays", Physics of plasmas, v. 22, pp. 122007, 2015.

[28] REUTER, S, VON WOEDTKE, T., WELTMANN, K-D., "The kINPen-a review on physics and chemistry of the atmospheric pressure plasma jet and its applications", J. Phys. D: Appl. Phys., v. 51, pp. 233001 (51pp), 2018.

[29] ZHOUA, Y., et al., "Investigation of discharge characteristics of DBD plasma produced with multineedle top plate electrodes in water by optical emission spectroscopy, Vacuum, v.162, pp. 121-127, 2019.

[30] WERTHEIMER, M.R., RADU, I., BARTNIKAS, R., "Dielectric barrier discharges (DBD) in gases at atmospheric pressure: effect of charge trapping", Proc. 12th Int. Symp. on Electrets, ISE-12, p. 231-234, 2005.

[31] FREITAS, R.C.S., VITORIANO,J.O., ALVES JUNIOR, C. "Investigating the aging of water treated by cold atmospheric plasma”, In: Anais do XL Congresso Brasileiro de Aplicações de Vácuo na Indústria e na Ciência, Guaratinguetá, SP, October 07-10, 2019.

[32] FREITAS, R.C.S., "Degradação do corante alaranjado de metila por plasma atmosférico" Trabalho de conclusão de curso, DCEN - UFERSA, Mossoró, RN-Brasil, 2019.

[33] BARAUNA, J. B. F.O., PEREIRA, C.S., GONÇALVES, I.A., et al., "Sodium Chloride Crystallization by Electric Discharge in Brine", Materials Research-Ibero-american Journal of Materials., v.6, pp.1 - 9, 2017.

[34] DE ALMADA, L. F. A., "Cristalização de flor de sal em soluções hipersalinas naturais induzida por evaporação em diferentes condições de umidade e plasma atmosférico", Dissertação de mestrado, PPGCEMUFERSA, Mossoró, RN-Brasil, Março/2020.

[35] FONTES, K.E.S., "Alternativas para extração de sais a partir de efluentes da indústria salineira sob diferentes condições de umidade e plasma atmosférico", Dissertação de mestrado, PPGCEM-UFERSA, Mossoró, RN-Brasil, Março/2020.

[36] GONÇALVES, I., BARAUNA, J.B.F.O., CUNHA-FILHO, F., et al., "Reduction of Aqueous Ag+ Steered by Electrochemical Plasma: Connecting the Bulk pH Variation with the Reaction Pathways for Hydrated Electrons", Journal of the Brazilian Chemical Society, , v.1, pp.1 - 14, 2019.

[37] F. E. P. DIÓGENES, "Emprego do plasma de descarga por barreira dielétrica (DBD) na inativação de fungos e na superação de dormência em sementes de Erythrina velutina Willd", Tese de doutorado, PPGFITO-UFERSA, Mossoró-RN, Brasil, Outubro/2017.

[38] FERNANDES, M. J., “Aplicação de plasma atmosférico em descarga por barreira dielétrica para descontaminação de água de coco”, Dissertação de mestrado, PPGCEM-UFERSA, Mossoró-RN, Brasil, Março/2020.

[39] Da Silva, A.R.M., Farias, M.L., Da Silva, D.L.S., et al., "Using atmospheric plasma to increase wettability, imbibition and germination of physically dormant seeds of Mimosa Caesalpiniafolia", Colloids and Surfaces B-Biointerfaces. , v.1, pp.1 - 10, 2017.

[40] ALVES JUNIOR, C., VITORIANO, J.O., DA SILVA, D.L.S., et al., "Water uptake mechanism and 
germination of Erythrina velutina seeds treated with atmospheric plasma", Scientific Reports., v.6, pp.33722 33729, 2016.

[41] ALVES-JUNIOR, C., DA SILVA, D. L. S., VITORIANO, J. O., et al., "The water path in plasmatreated Leucaena seeds. Seed Science Research., v.3, p.1 - 8, 2020.

[42] ANGELIS, S.S., CAMPELO, M.C.S., REBOUÇAS, L.O.S., et al., "Use of cold atmospheric plasma to preserve the quality of white shrimp Litopenaeus vannamei", Journal of Food Protection., v.82, p.1217 $1223,2019$.

[43] ALVES JÚNIOR, C., PEREIRA, T. T. DE O., MELO, R. R., et al., "Características elétricas e eficiência energética de um sistema de descarga de barreira dielétrica", Revista Brasileira de Aplicações do Vácuo on Line, v.36, pp.107 - 112, 2018.

\section{ORCID}

Clodomiro Alves Junior

https://orcid.org/0000-0002-5547-5922 